\title{
Three-Dimensional Coupled NLS Equations for Envelope Gravity Solitary Waves in Baroclinic Atmosphere and Modulational Instability
}

\author{
Baojun Zhao $\mathbb{D}$, ${ }^{1,2}$ Ruyun Wang $\mathbb{D}^{1},{ }^{1}$ and Hongwei Yang $\mathbb{D}^{3,4}$ \\ ${ }^{1}$ College of Oceanography, Hohai University, Nanjing 210098, China \\ ${ }^{2}$ College of Roads and Bridges, Nanjing Vocational Institute of Transport Technology, Nanjing 211188, China \\ ${ }^{3}$ College of Mathematics and Systems Science, Shandong University of Science and Technology, Qingdao, Shandong 266590, China \\ ${ }^{4}$ Key Laboratory of Meteorological Disaster of Ministry of Education, Nanjing University of Information Science and Technology, \\ Nanjing 210044, China
}

Correspondence should be addressed to Ruyun Wang; wangry@hhu.edu.cn

Received 3 August 2017; Accepted 30 October 2017; Published 8 January 2018

Academic Editor: Qin Yuming

Copyright (c) 2018 Baojun Zhao et al. This is an open access article distributed under the Creative Commons Attribution License, which permits unrestricted use, distribution, and reproduction in any medium, provided the original work is properly cited.

\begin{abstract}
Envelope gravity solitary waves are an important research hot spot in the field of solitary wave. And the weakly nonlinear model equations system is a part of the research of envelope gravity solitary waves. Because of the lack of technology and theory, previous studies tried hard to reduce the variable numbers and constructed the two-dimensional model in barotropic atmosphere and could only describe the propagation feature in a direction. But for the propagation of envelope gravity solitary waves in real ocean ridges and atmospheric mountains, the three-dimensional model is more appropriate. Meanwhile, the baroclinic problem of atmosphere is also an inevitable topic. In the paper, the three-dimensional coupled nonlinear Schrödinger (CNLS) equations are presented to describe the evolution of envelope gravity solitary waves in baroclinic atmosphere, which are derived from the basic dynamic equations by employing perturbation and multiscale methods. The model overcomes two disadvantages: (1) baroclinic problem and (2) propagation path problem. Then, based on trial function method, we deduce the solution of the CNLS equations. Finally, modulational instability of wave trains is also discussed.
\end{abstract}

\section{Introduction}

According to media reported, "6.1 the Oriental Star cruise ship capsized event" was caused by the violence rainstorm attack which was brought by a sudden squall lines process. Unfortunately, the forecast for the severe weather such as squall lines is very difficult. As we know, the nonlinearity concentration of the gravity waves makes the energy assemble together and forms disastrous weather phenomena, such as squall lines and rainstorm. So constructing the theoretical model of gravity waves suits the real atmosphere condition and analyzing the formation mechanism of disastrous weather phenomena based on the theoretical model has significant scientific meaning and application value.

The first discovery of solitary waves was attributed to Russell [1]; then, the study of solitary wave continued to deepen. There was vast research literature devoted to the solitary waves. Solitary waves in the westerly shear flow were first found by Long [2]. Afterward, Benny [3] amplified the conclusions and got a conclusion that velocity and amplitude of solitary waves were related. Recently, a variety of equation models which described the solitary waves, such as ILWBurgers equation and ZK-Burgers equation, were discussed by Yang et al. [4, 5]. Meanwhile the generation and evolution of solitary waves in different topography condition and different fluid depths were discussed. In recent years, in the solitary waves community, gravity solitary wave as a rising star had been paid more and more attention by many scientists. In the 60s, Long [2] proposed that the amplitude of atmospheric gravity wave satisfied KdV equation and got the solutions of amplitude. Then a lot of researchers obtained nonlinear $\mathrm{KdV}$ equations of gravity waves from the basic 
dynamic equation. From the original equation of two layers of $f$-plane, under the shearing basic flow, the famous KdV equation was derived out by $\mathrm{Li}$ [6], and he pointed out that the nonlinear characteristic of gravity waves was the generation mechanism of squall lines. S. K. Liu and S. D. Liu [7] based on nonlinear atmospheric motion equation deduced out nonlinear gravity wave solutions and found that gravitational wave amplitude with propagation speed was proportional. Later, Boussinesq equation was derived in Luo [8] to describe the algebraic gravity solitary wave in atmosphere. Additionally, two-dimensional dissipative nonlinear Schrödinger equation [9] was also obtained to reflect the evolution of envelope solitary Rossby waves.

Therefore, for better understanding the characteristic of solitary waves deeply, it is desired to get the exact solutions $[10,11]$ of soliton equations. Up to now, people have obtained several sorts of nonlinear evolution equations and studied their properties, including the boundary value problem [1214], Hamiltonian structure $[15,16]$, integrable systems [17, 18], and conservation law [19]. In the process of solving nonlinear evolution equations, many solution methods are found, like Darboux transformation method [20-22], Hirota method [23], homogeneous balance method [24], Jacobi elliptic function method [25], symmetry method [26], trial function method [27], the alternative variational-asymptotic method [28-31], and so on [32,33]. Biswas et al. [34-36] have also found some other methods of solving the nonlinear partial equation, but we noticed that they have not been applied to solve the three-dimensional CNLS equations.

Through the analysis of previous research on the gravity wave we can get the following two points:

(1) In order to simplify the calculation, the former researches studied the propagation of gravity solitary waves in two-dimension space and barotropic atmosphere [37]. But for the envelope of gravity waves in the real atmosphere, it was not sufficient to consider only two dimensions. And the initial equation was a simple model in barotropic atmosphere

$$
\begin{array}{r}
\left(\frac{\partial}{\partial t}+u \frac{\partial}{\partial x}+v \frac{\partial}{\partial y}\right) u-v=-\frac{\partial \phi}{\partial x}, \\
\left(\frac{\partial}{\partial t}+u \frac{\partial}{\partial x}+v \frac{\partial}{\partial y}\right) v+u=-\frac{\partial \phi}{\partial y}, \\
\left(\frac{\partial}{\partial t}+u \frac{\partial}{\partial x}+v \frac{\partial}{\partial y}\right) \phi+\lambda^{-2}\left(\frac{\partial u}{\partial x}+\frac{\partial v}{\partial y}\right)=0,
\end{array}
$$

where $\lambda=L / L_{0}, L_{0}=c_{0}^{*} / f_{0}, c_{0}^{*}=g^{*} H_{0}$, and $g^{*}=g((\rho-$ $\left.\varrho^{\prime}\right) / \rho$ ). These variables were simplified. Therefore, the threedimensional model in baroclinic atmosphere is more in line with the actual atmospheric conditions.

(2) Previous studies have focused on the solution of gravity waves and the dynamic characteristics of the wave equation mainly based on the numerical calculation from the original equation. As we all know, many of the variables in the original equation are simplified and dimensional; therefore, numerical simulation directly based on the original equation is usually imprecise and difficult. So it is necessary for us to find the appropriate model and solve it to study the evolution of the waves.
In this paper, overcoming the limitations of calculations and using the appropriate method we obtain a new model. The paper will be organized as follows: (1) using multiscale analysis and turbulence method, from the basic dynamic equations of multivariable in baroclinic environment, we derived out the gravity wave model in Section 2, which is a coupled nonlinear Schrödinger equation (CNLS). Not only is the model three-dimensional and more suited to describe the feature of two envelope gravity solitary waves in a plane, specially, but also it is a coupled model and can show the interaction process between two waves. (2) Based on the CNLS model, using the trial function method to solve the equations, the analytical solution was obtained in Section 3. By observing the structures of the solution, the evolution characteristics of gravity solitary waves was obtained in Section 4. (3) Finally, modulational instability of a uniform three-dimensional gravity waves trains was also discussed.

\section{Derivation of the Three-Dimensional CNLS Equations Group}

Using the sum of disturbance pressure gradient force and buoyancy force, express the vertical pressure gradient force and gravity force, adopt Boussinesq approximation, and the basic dynamic equations of atmospheric motion are as follows:

$$
\begin{aligned}
\frac{\partial u}{\partial t}+u \frac{\partial u}{\partial x}+v \frac{\partial u}{\partial y}+w \frac{\partial u}{\partial z} & =-\frac{1}{\rho_{0}} \frac{\partial p}{\partial x}+f v \\
\frac{\partial v}{\partial t}+u \frac{\partial v}{\partial x}+v \frac{\partial v}{\partial y}+w \frac{\partial v}{\partial z} & =-\frac{1}{\rho_{0}} \frac{\partial p}{\partial y}-f u \\
\frac{\partial w}{\partial t}+u \frac{\partial w}{\partial x}+v \frac{\partial w}{\partial y}+w \frac{\partial w}{\partial z} & =-\frac{1}{\rho_{0}} \frac{\partial p}{\partial z}+\frac{g \theta}{\theta_{0}}, \\
\frac{\partial \theta}{\partial t}+u \frac{\partial \theta}{\partial x}+v \frac{\partial \theta}{\partial y}+\sigma w & =0 \\
\frac{\partial\left(\rho_{0} u\right)}{\partial x}+\frac{\partial\left(\rho_{0} v\right)}{\partial y}+\frac{\partial\left(\rho_{0} w\right)}{\partial z} & =0
\end{aligned}
$$

in the above equations, $\theta_{0}$ is the temperature of environmental flow field and $\rho_{0}$ is the density; $\sigma=d \theta_{0} / d z$. Each variable above will be dimensionless. Let

$$
\begin{aligned}
(x, y) & =L\left(x^{\prime}, y^{\prime}\right), \\
z & =D\left(z^{\prime}\right), \\
t & =f^{-1}\left(t^{\prime}\right), \\
(u, v) & =U\left(u^{\prime}, v^{\prime}\right), \\
w & =\frac{U}{L} D\left(w^{\prime}\right), \\
\theta & =\delta \theta\left(\theta^{\prime}\right),
\end{aligned}
$$




$$
\begin{aligned}
\delta p_{x, y} & =\frac{P}{g H} f L U\left(p^{\prime}\right), \\
\delta p_{z} & =\frac{P}{\theta_{0} H} \delta \theta\left(p^{\prime}\right), \\
\rho_{0} & =\frac{P}{g H}\left(\rho_{s}\right),
\end{aligned}
$$

$$
\begin{aligned}
& \frac{\partial \theta^{\prime}}{\partial t^{\prime}}+u^{\prime} \frac{\partial \theta^{\prime}}{\partial x^{\prime}}+v^{\prime} \frac{\partial \theta^{\prime}}{\partial y^{\prime}}+w^{\prime}=0, \\
& \frac{\partial\left(\rho_{s} u^{\prime}\right)}{\partial x^{\prime}}+\frac{\partial\left(\rho_{s} v^{\prime}\right)}{\partial y^{\prime}}+\frac{\partial\left(\rho_{s} w^{\prime}\right)}{\partial z^{\prime}}=0 .
\end{aligned}
$$

where $H$ is the height of the homogeneous atmosphere, $P$ is the characteristics pressure of the ground, $\delta p_{x, y}$ is the horizontal pressure changes, and $\delta p_{z}$ is the pressure changes in the vertical direction. Substituting (3) into (2), get the dimensionless equations as follows:

$$
\begin{aligned}
& \frac{\partial u^{\prime}}{\partial t^{\prime}}+\frac{U}{f L}\left(u^{\prime} \frac{\partial u^{\prime}}{\partial x^{\prime}}+v^{\prime} \frac{\partial u^{\prime}}{\partial y^{\prime}}+w^{\prime} \frac{\partial u^{\prime}}{\partial z^{\prime}}\right)=-\frac{1}{\rho_{s}} \frac{\partial p^{\prime}}{\partial x^{\prime}}+v^{\prime}, \\
& \frac{\partial v^{\prime}}{\partial t^{\prime}}+\frac{U}{f L}\left(u^{\prime} \frac{\partial v^{\prime}}{\partial x^{\prime}}+v^{\prime} \frac{\partial v^{\prime}}{\partial y^{\prime}}+w^{\prime} \frac{\partial v^{\prime}}{\partial z^{\prime}}\right)=-\frac{1}{\rho_{s}} \frac{\partial p^{\prime}}{\partial y^{\prime}}-u^{\prime}, \\
& \frac{\partial w^{\prime}}{\partial t^{\prime}}+\frac{U}{f L}\left(u^{\prime} \frac{\partial w^{\prime}}{\partial x^{\prime}}+v^{\prime} \frac{\partial w^{\prime}}{\partial y^{\prime}}+w^{\prime} \frac{\partial w^{\prime}}{\partial z^{\prime}}\right) \\
& \quad=\frac{g L \delta \theta}{D f U \theta_{0}}\left(-\frac{1}{\rho_{s}} \frac{\partial p^{\prime}}{\partial z^{\prime}}+\theta^{\prime}\right), \\
& \frac{\partial \theta^{\prime}}{\partial t^{\prime}}+\frac{U}{f L}\left(u^{\prime} \frac{\partial \theta^{\prime}}{\partial x^{\prime}}+v^{\prime} \frac{\partial \theta^{\prime}}{\partial y^{\prime}}\right)+\frac{\sigma U D}{f L \delta \theta} w^{\prime}=0, \\
& \frac{\partial\left(\rho_{s} u^{\prime}\right)}{\partial x^{\prime}}+\frac{\partial\left(\rho_{s} v^{\prime}\right)}{\partial y^{\prime}}+\frac{\partial\left(\rho_{s} w^{\prime}\right)}{\partial z^{\prime}}=0,
\end{aligned}
$$

supposing $D \sim H$ in the above. Because the second term of the left side of the fourth formula is lesser, get the following approximate:

$$
\begin{aligned}
& \delta \theta \sim \frac{\sigma U D}{f L}, \\
& \frac{U}{f L} \sim o(1),
\end{aligned}
$$

introducing parameter $\varepsilon=f^{2} / N^{2}(\varepsilon \ll 1)$, where $N^{2}=$ $g \theta / \theta_{0}$. By varying, (4) transforms to the following:

$$
\begin{aligned}
& \frac{\partial u^{\prime}}{\partial t^{\prime}}+u^{\prime} \frac{\partial u^{\prime}}{\partial x^{\prime}}+v^{\prime} \frac{\partial u^{\prime}}{\partial y^{\prime}}+w^{\prime} \frac{\partial u^{\prime}}{\partial z^{\prime}}=-\frac{1}{\rho_{s}} \frac{\partial p^{\prime}}{\partial x^{\prime}}+v^{\prime}, \\
& \frac{\partial v^{\prime}}{\partial t^{\prime}}+u^{\prime} \frac{\partial v^{\prime}}{\partial x^{\prime}}+v^{\prime} \frac{\partial v^{\prime}}{\partial y^{\prime}}+w^{\prime} \frac{\partial v^{\prime}}{\partial z^{\prime}}=-\frac{1}{\rho_{s}} \frac{\partial p^{\prime}}{\partial y^{\prime}}-u^{\prime}, \\
& \frac{\partial w^{\prime}}{\partial t^{\prime}}+u^{\prime} \frac{\partial w^{\prime}}{\partial x^{\prime}}+v^{\prime} \frac{\partial w^{\prime}}{\partial y^{\prime}}+w^{\prime} \frac{\partial w^{\prime}}{\partial z^{\prime}} \\
& =\epsilon^{-1}\left(-\frac{1}{\rho_{s}} \frac{\partial p^{\prime}}{\partial x^{\prime}}+\theta^{\prime}\right),
\end{aligned}
$$

Introduction of multiscale variables (omit the sign at the top right corner of the variables)

$$
\begin{gathered}
T_{1}=\epsilon t, \\
T_{2}=\epsilon^{2} t, \\
X_{1}=\epsilon x, \\
X_{2}=\epsilon^{2} x, \\
Y=\epsilon y,
\end{gathered}
$$

so long time and space scales are defined as

$$
\begin{aligned}
\frac{\partial}{\partial t} & =\frac{\partial}{\partial t}+\varepsilon \frac{\partial}{\partial T_{1}}+\varepsilon^{2} \frac{\partial}{\partial T_{2}} \\
\frac{\partial}{\partial x} & =\frac{\partial}{\partial x}+\varepsilon \frac{\partial}{\partial X_{1}}+\varepsilon^{2} \frac{\partial}{\partial X_{2}} \\
\frac{\partial}{\partial y} & =\frac{\partial}{\partial y}+\varepsilon \frac{\partial}{\partial Y} \\
\frac{\partial}{\partial z} & =\frac{\partial}{\partial z}
\end{aligned}
$$

$u^{\prime}, v^{\prime}, w^{\prime}, p^{\prime}, \theta^{\prime}$ in (6) were expanded according to the small parameter $\varepsilon$ :

$$
\begin{aligned}
& u^{\prime}=U(y, z)+\epsilon\left(u_{0}+\epsilon u_{1}+\epsilon^{2} u_{2}\right)+\cdots, \\
& v^{\prime}=V(y, z)+\epsilon\left(v_{0}+\epsilon v_{1}+\epsilon^{2} v_{2}\right)+\cdots, \\
& w^{\prime}=\epsilon\left(w_{0}+\epsilon w_{1}+\epsilon^{2} w_{2}\right)+\cdots, \\
& \theta^{\prime}=\Theta(y, z)+\epsilon\left(\theta_{0}+\epsilon \theta_{1}+\epsilon^{2} \theta_{2}\right)+\cdots, \\
& p^{\prime}=P(y, z)+\epsilon\left(p_{0}+\epsilon p_{1}+\epsilon^{2} p_{2}\right)+\cdots,
\end{aligned}
$$

where $U, V, P, \Theta$ are the functions of $y, z$, where $U$ is the speed of the basic flow, $P$ is the air pressure, and $\Theta$ is the temperature field.

Substituting (8) and (9) into (6), the zero-order approximation of $\varepsilon$ can be obtained: 


$$
O\left(\epsilon^{0}\right):\left\{\begin{array}{l}
-\frac{1}{\rho_{s}} \frac{\partial P}{\partial y}-U=0 \\
-\frac{1}{\rho_{s}} \frac{\partial P}{\partial z}+\Theta=0
\end{array}\right.
$$

It is shown that basic flow is geostrophic equilibrium and static balance. For a basic flow we can further assume that

$$
\left|\frac{1}{\rho_{s}^{2}} \frac{\partial \rho_{s}}{\partial y}\right| \ll 1
$$

in the first formula in (10), derivation of $z$, and the second formula in (10), derivation of $y$, so that

$$
\frac{\partial U}{\partial z}=-\frac{\partial \Theta}{\partial y}
$$

Further, take the first-order approximation of $\varepsilon$ and introduce the new variables

$$
\begin{gathered}
\rho_{s} u_{0}=u_{0}, \\
\rho_{s} v_{0}=v_{0}, \\
\rho_{s} w_{0}=w_{0}, \\
\rho_{s} \theta_{0}=\theta_{0} ;
\end{gathered}
$$

we have

$$
O\left(\epsilon^{1}\right):
$$

$$
\left\{\begin{array}{l}
\frac{\partial u_{0}}{\partial t}+U \frac{\partial u_{0}}{\partial x}+V \frac{\partial u_{0}}{\partial y}+\left(U_{y}-1\right) v_{0}+U_{z} w_{0}+\frac{\partial p_{0}}{\partial x}=0 \\
\frac{\partial v_{0}}{\partial t}+U \frac{\partial v_{0}}{\partial x}+V \frac{\partial v_{0}}{\partial y}+\frac{\partial p_{0}}{\partial y}+U_{0}=0 \\
\frac{\partial p_{0}}{\partial z}-\theta_{0}=0 \\
\frac{\partial \theta_{0}}{\partial t}+U \frac{\partial \theta_{0}}{\partial x}+V \frac{\partial \theta_{0}}{\partial y}+\Theta_{y} v_{0}+w_{0}=0 \\
\frac{\partial u_{0}}{\partial x}+\frac{\partial v_{0}}{\partial y}+\frac{\partial w_{0}}{\partial z}=0
\end{array}\right.
$$

After eliminating other variables in (14), we can get the equation about $p_{0}$, as follows:

$$
L_{y, z}\left(\frac{\partial p_{0}}{\partial x}\right)=0
$$

where

$$
\begin{aligned}
\Omega= & U_{y}-1+U_{z}^{2}, \\
\Omega_{y}= & \frac{\partial \Omega}{\partial y}, \\
\Omega_{z}= & \frac{\partial \Omega}{\partial z}, \\
L_{y, z}= & \frac{\partial^{2}}{\partial y^{2}}-\left(U_{y}-1\right) \frac{\partial^{2}}{\partial z^{2}}+2 U_{z} \frac{\partial^{2}}{\partial y \partial z} \\
& +\left[U_{z z}-\frac{\Omega_{y}}{\Omega}-U_{z} \frac{\Omega_{z}}{\Omega}\right] \frac{\partial}{\partial y} \\
& +\left[\left(U_{y}-1\right) \frac{\Omega_{z}}{\Omega}-U_{z} \frac{\Omega_{y}}{\Omega}\right] \frac{\partial}{\partial z} \\
& -\frac{1}{U}\left[U_{z z}-\frac{\Omega_{y}}{\Omega}-U_{z} \frac{\Omega_{z}}{\Omega}\right] .
\end{aligned}
$$

Clearly, (15) is a variable separable equation; assume its solution is

$$
\begin{array}{r}
p_{0}=\sum_{j=1}^{2} \widetilde{p_{0 j}}(y, z) A_{j}\left(T_{1}, T_{2}, X_{1}, X_{2}, Y\right) e^{i\left(k_{j} x-\omega_{j} t\right)}, \\
(j=1,2) ;
\end{array}
$$

under a certain definite solution condition, we can get $\widetilde{p_{0 j}}$; further, all the solutions of (14) can be obtained:

$$
u_{0}=\sum_{j=1}^{2} \widetilde{u_{0 j}}(y, z) A_{j}\left(T_{1}, T_{2}, X_{1}, X_{2}, Y\right) e^{i\left(k_{j} x-\omega_{j} t\right)}
$$

$$
\begin{aligned}
& v_{0}=\sum_{j=1}^{2} \widetilde{v_{0 j}}(y, z) A_{j}\left(T_{1}, T_{2}, X_{1}, X_{2}, Y\right) e^{i\left(k_{j} x-\omega_{j} t\right)} \\
& w_{0}=\sum_{j=1}^{2} \widetilde{w_{0 j}}(y, z) A_{j}\left(T_{1}, T_{2}, X_{1}, X_{2}, Y\right) e^{i\left(k_{j} x-\omega_{j} t\right)} \\
& \theta_{0}=\sum_{j=1}^{2} \widetilde{\theta_{0 j}}(y, z) A_{j}\left(T_{1}, T_{2}, X_{1}, X_{2}, Y\right) e^{i\left(k_{j} x-\omega_{j} t\right)} .
\end{aligned}
$$

Taking the second-order approximation of $\varepsilon$ and introducing the new variables

$$
\begin{gathered}
\rho_{s} u_{1}=u_{1}, \\
\rho_{s} v_{1}=v_{1}, \\
\rho_{s} w_{1}=w_{1}, \\
\rho_{s} \theta_{1}=\theta_{1},
\end{gathered}
$$

we have 
$O\left(\epsilon^{2}\right):$

$$
\left\{\begin{array}{l}
\frac{\partial u_{1}}{\partial t}+U \frac{\partial u_{1}}{\partial x}+V \frac{\partial u_{1}}{\partial y}+\left(U_{y}-1\right) v_{1}+U_{z} w_{1}+\frac{\partial p_{1}}{\partial x}=-\left[\frac{\partial u_{0}}{\partial T_{1}}+U \frac{\partial u_{0}}{\partial X_{1}}+V \frac{\partial u_{0}}{\partial Y}+\frac{1}{\rho_{s}}\left(u_{0} \frac{\partial u_{0}}{\partial x}+v_{0} \frac{\partial u_{0}}{\partial y}+w_{0} \frac{\partial u_{0}}{\partial z}\right)+\frac{\partial p_{0}}{\partial X_{1}}\right] \\
\frac{\partial v_{1}}{\partial t}+U \frac{\partial v_{1}}{\partial x}+V \frac{\partial v_{1}}{\partial y}+u_{1}+V_{y} v_{1}+V_{z} w_{1}+\frac{\partial p_{1}}{\partial y}=-\left[\frac{\partial v_{0}}{\partial T_{1}}+U \frac{\partial v_{0}}{\partial X_{1}}+V \frac{\partial v_{0}}{\partial Y}+\frac{1}{\rho_{s}}\left(u_{0} \frac{\partial v_{0}}{\partial x}+v_{0} \frac{\partial v_{0}}{\partial y}+w_{0} \frac{\partial v_{0}}{\partial z}\right)+\frac{\partial p_{0}}{\partial Y}\right], \\
\frac{\partial p_{1}}{\partial z}-\theta_{1}=-\left(\frac{\partial w_{0}}{\partial t}+U \frac{\partial w_{0}}{\partial x}+V \frac{\partial w_{0}}{\partial y}\right), \\
\frac{\partial \theta_{1}}{\partial t}+U \frac{\partial \theta_{1}}{\partial x}+V \frac{\partial \theta_{1}}{\partial y}+\Theta_{y} v_{1}+w_{1}=-\left[\frac{\partial \theta_{0}}{\partial T_{1}}+U \frac{\partial \theta_{0}}{\partial X_{1}}+V \frac{\partial \theta_{0}}{\partial Y}+\frac{1}{\rho_{s}}\left(u_{0} \frac{\partial \theta_{0}}{\partial x}+v_{0} \frac{\partial \theta_{0}}{\partial y}\right)\right], \\
\frac{\partial u_{1}}{\partial x}+\frac{\partial v_{1}}{\partial y}+\frac{\partial w_{1}}{\partial z}=-\left(\frac{\partial u_{0}}{\partial X_{1}}+\frac{\partial v_{0}}{\partial Y}\right) ;
\end{array}\right.
$$

let

$$
\begin{aligned}
A u_{1}= & -\frac{\partial u_{0}}{\partial T_{1}}-U \frac{\partial u_{0}}{\partial X_{1}}-V \frac{\partial u_{0}}{\partial Y} \\
& -\frac{1}{\rho_{s}}\left(u_{0} \frac{\partial u_{0}}{\partial x}+v_{0} \frac{\partial u_{0}}{\partial y}+w_{0} \frac{\partial u_{0}}{\partial z}\right)-\frac{\partial p_{0}}{\partial X_{1}}, \\
A v_{1}= & -\frac{\partial v_{0}}{\partial T_{1}}-U \frac{\partial v_{0}}{\partial X_{1}}-V \frac{\partial v_{0}}{\partial Y} \\
& -\frac{1}{\rho_{s}}\left(u_{0} \frac{\partial v_{0}}{\partial x}+v_{0} \frac{\partial v_{0}}{\partial y}+w_{0} \frac{\partial v_{0}}{\partial z}\right)-\frac{\partial p_{0}}{\partial Y} \\
A w_{1}= & -\frac{\partial w_{0}}{\partial t}-U \frac{\partial w_{0}}{\partial x}-V \frac{\partial w_{0}}{\partial y} \\
A \theta_{1}= & -\frac{\partial \theta_{0}}{\partial T_{1}}-U \frac{\partial \theta_{0}}{\partial X_{1}}-V \frac{\partial \theta_{0}}{\partial Y} \\
& -\frac{1}{\rho_{s}}\left(u_{0} \frac{\partial \theta_{0}}{\partial x}+v_{0} \frac{\partial \theta_{0}}{\partial y}\right) \\
A p_{1}= & -\frac{\partial u_{0}}{\partial X_{1}}-\frac{\partial v_{0}}{\partial Y} .
\end{aligned}
$$

Substitute (18) into (21)

$A u_{1}$

$$
\begin{aligned}
& =\sum_{j=1}^{2}-\left[\widetilde{u_{0 j}} A_{j T_{1}}+\left(U \widetilde{u_{0}}+\widetilde{p_{0 j}}\right) A_{j X_{1}}+V \widetilde{u_{0 j}} A_{j Y}\right] \\
& \cdot e^{i\left(k_{j} x-\omega_{j} t\right)}-\frac{1}{\rho_{s}}\left(i k \widetilde{u_{0 j}}{ }^{2}+\widetilde{v_{0 j}} \widetilde{u_{0 j}}+\widetilde{w_{0 j}} \widetilde{u_{0 j}}\right)\left|A_{j}\right|^{2} \\
& \cdot e^{2 i\left(k_{j} x-w_{j} t\right)}
\end{aligned}
$$

$A v_{1}$

$$
\begin{aligned}
& =\sum_{j=1}^{2}-\left[\widetilde{v_{0 j}} A_{j T_{1}}+U \widetilde{v_{0 j}} A_{j X_{1}}+\left(V \widetilde{v_{0 j}}+\widetilde{p_{0 j}}\right) A_{j Y}\right] \\
& \cdot e^{i\left(k_{j} x-\omega_{j} t\right)}-\frac{1}{\rho_{s}}\left(i k \widetilde{u_{0 j}} \widetilde{v_{0 j}}+\widetilde{v_{0 j}} \widetilde{v_{0 j}}+\widetilde{w_{0 j}} V_{z}\right)\left|A_{j}\right|^{2} \\
& \cdot e^{2 i\left(k_{j} x-w_{j} t\right)}
\end{aligned}
$$$$
A w_{1}=\sum_{j=1}^{2}\left[i \widetilde{w_{0 j}}\left(\omega_{j}-k_{j} U\right)-V \widetilde{w_{0 j_{y}}}\right] A_{j} e^{i\left(K_{j} x-\omega_{j} t\right)},
$$$$
A \theta_{1}=\sum_{j=1}^{2}-\left[\widetilde{\theta_{0 j}} A_{j T_{1}}+U \widetilde{\theta_{0 j}} A_{j X_{1}}+V \widetilde{\theta_{0 j}} A_{j Y}\right]
$$$$
\cdot e^{i\left(k_{j} x-\omega_{j} t\right)}-\frac{1}{\rho_{s}}\left(i k \widetilde{u_{0 j}} \widetilde{\theta_{0 j}}+\widetilde{v_{0 j}} \widetilde{\theta_{0 j}}\right)\left|A_{j}\right|^{2}
$$$$
\cdot e^{2 i\left(k_{j} x-w_{j} t\right)},
$$$$
A p_{1}=\sum_{j=1}^{2}-\left(\widetilde{u_{0 j}} A_{j X_{1}}+\widetilde{v_{0 j}} A_{j Y}\right) e^{i\left(k_{j} x-\omega_{j}\right) t} .
$$

After eliminating other variables in (20), the equation about $p_{1}$ can be obtained as follows:

$$
\begin{aligned}
L_{y, z}\left(\frac{\partial p_{1}}{\partial x}\right)= & L_{1 y, z}\left(A u_{1}\right)+L_{2 y, z}\left(A v_{1}\right) \\
& +L_{3 y, z}\left(A w_{1}\right)+L_{4 y, z}\left(A \theta_{1}\right)
\end{aligned}
$$

where

$$
\begin{aligned}
& L_{1 y, z}=\frac{\partial}{\partial y}+U_{z z}+U_{z} \frac{\partial}{\partial z}+\frac{1}{U}+\frac{1}{\Omega}\left(\Omega_{y}+U_{z} \Omega_{z}\right), \\
& L_{2 y, z}=-\frac{1}{U}\left[\frac{\partial}{\partial y}+U_{z z}+U_{z} \frac{\partial}{\partial z}-\frac{1}{\Omega}\left(\Omega_{y}+U_{z} \Omega_{z}\right)\right],
\end{aligned}
$$




$$
\begin{aligned}
L_{3 y, z} & =\frac{1}{U}\left[U_{z} \frac{\partial}{\partial y}-\left(U_{y}-1\right) \frac{\partial}{\partial z}-\frac{1}{\Omega} U_{z} \Omega_{y}\right. \\
- & \left.\frac{1}{\Omega}\left(U_{y}-1\right) \Omega_{z}\right], \\
L_{4 y, z} & =U_{z} \frac{\partial}{\partial y}+\frac{U_{z}}{U}-\left(U_{y}-1\right) \frac{\partial}{\partial z}-\frac{\Omega_{y}}{\Omega} U_{z} \\
- & \frac{\left(U_{y}-1\right)}{U} \frac{\Omega_{z}}{\Omega} .
\end{aligned}
$$

Similarly, (23) is also a variable separable equation; with comparison on both ends of (23), assume its basic solution is

$$
p_{1}=\sum_{j=1}^{2} \widetilde{p_{1 j}}(y, z)\left[A_{j} e^{i\left(k_{j} x-\omega_{j} t\right)}+\left|A_{j}\right|^{2} e^{2 i\left(k_{j} x-w_{j} t\right)}\right]
$$

further, we can get all solutions of (23):

$$
\begin{aligned}
& u_{1}=\sum_{j=1}^{2} \widetilde{u_{1 j}}\left[A_{X_{1}} e^{i\left(k_{j} x-\omega_{j} t\right)}+\left|A_{j}\right|^{2} e^{2 i\left(k_{j} x-\omega_{j} t\right)}\right], \\
& v_{1}=\sum_{j=1}^{2} \widetilde{v_{1 j}}\left[A_{Y} e^{i\left(k_{j} x-\omega_{j} t\right)}+\left|A_{j}\right|^{2} e^{2 i\left(k_{j} x-\omega_{j} t\right)}\right], \\
& w_{1}=\sum_{j=1}^{2} \widetilde{w_{1 j}}\left[A_{Y} e^{i\left(k_{j} x-\omega_{j} t\right)}+\left|A_{j}\right|^{2} e^{2 i\left(k_{j} x-w_{j} t\right)}\right], \\
& \theta_{1}=\sum_{j=1}^{2} \widetilde{\theta_{1 j}}\left[A_{X_{1}} e^{i\left(k_{j} x-\omega_{j} t\right)}+\left|A_{j}\right|^{2} e^{2 i\left(k_{j} x-w_{j} t\right)}\right] .
\end{aligned}
$$

Similar to the above, introducing the new variables,

$$
\begin{gathered}
\rho_{s} u_{2}=u_{2}, \\
\rho_{s} v_{2}=v_{2}, \\
\rho_{s} w_{2}=w_{2}, \\
\rho_{s} \theta_{2}=\theta_{2},
\end{gathered}
$$

the third-order approximation of $\varepsilon$ transforms to

$O\left(\epsilon^{3}\right):$

$$
\left\{\begin{array}{l}
\frac{\partial u_{2}}{\partial t}+U \frac{\partial u_{2}}{\partial x}+V \frac{\partial u_{2}}{\partial y}+\left(U_{y}-1\right) v_{2}+U_{z} w_{2}+\frac{\partial p_{2}}{\partial x} \\
=-\left[\frac{\partial u_{0}}{\partial T_{2}}+U \frac{\partial u_{0}}{\partial X_{2}}+U \frac{\partial u_{1}}{\partial X_{1}}+V \frac{\partial u_{1}}{\partial Y}+\frac{\partial u_{1}}{\partial T_{1}}+\frac{1}{\rho_{s}}\left(u_{0} \frac{\partial u_{0}}{\partial X_{1}}+v_{0} \frac{\partial u_{0}}{\partial Y}+u_{1} \frac{\partial u_{0}}{\partial x}+v_{1} \frac{\partial u_{0}}{\partial y}+w_{1} \frac{\partial u_{0}}{\partial z}+u_{0} \frac{\partial u_{1}}{\partial x}+v_{0} \frac{\partial u_{1}}{\partial y}+w_{0} \frac{\partial u_{1}}{\partial z}\right)+\frac{\partial p_{0}}{\partial X_{2}}+\frac{\partial p_{1}}{\partial X_{1}}\right], \\
\frac{\partial v_{2}}{\partial t}+U \frac{\partial v_{2}}{\partial x}+V \frac{\partial v_{2}}{\partial y}+V_{y} v_{2}+V_{z} w_{2}+\frac{\partial p_{2}}{\partial y}+u_{2}=-\left[\frac{\partial v_{0}}{\partial T_{2}}+U \frac{\partial v_{0}}{\partial X_{2}}+U \frac{\partial v_{1}}{\partial X_{1}}+V \frac{\partial v_{1}}{\partial Y}+\frac{\partial v_{1}}{\partial T_{1}}+\frac{1}{\rho_{s}}\left(u_{0} \frac{\partial v_{0}}{\partial X_{1}}+v_{0} \frac{\partial v_{0}}{\partial Y}+u_{1} \frac{\partial v_{0}}{\partial x}+v_{1} \frac{\partial v_{0}}{\partial y}+w_{1} \frac{\partial v_{0}}{\partial z}+u_{0} \frac{\partial v_{1}}{\partial x}+v_{0} \frac{\partial v_{1}}{\partial y}+w_{0} \frac{\partial v_{1}}{\partial z}\right)+\frac{\partial p_{1}}{\partial Y}\right] \\
\frac{\partial p_{2}}{\partial z}-\theta_{2}=-\left[\frac{\partial w_{0}}{\partial t_{1}}+U \frac{\partial w_{0}}{\partial X_{1}}+V \frac{\partial w_{0}}{\partial Y}+U \frac{\partial w_{1}}{\partial x}+\frac{1}{\rho_{s}}\left(u_{0} \frac{\partial w_{1}}{\partial x}+v_{0} \frac{\partial w_{1}}{\partial y}+w_{0} \frac{\partial w_{1}}{\partial z}\right)+\frac{\partial w_{1}}{\partial t}\right], \\
\frac{\partial \theta_{2}}{\partial t}+U \frac{\partial \theta_{2}}{\partial x}+V \frac{\partial \theta_{2}}{\partial y}+\Theta_{y} v_{2}+w_{2}=-\left[\frac{\partial \theta_{0}}{\partial T_{2}}+U \frac{\partial \theta_{0}}{\partial X_{2}}+U \frac{\partial \theta_{1}}{\partial X_{1}}+V \frac{\partial \theta_{1}}{\partial Y}+\frac{\partial \theta_{1}}{\partial T_{1}}+\frac{1}{\rho_{s}}\left(u_{0} \frac{\partial \theta_{0}}{\partial x_{1}}+v_{0} \frac{\partial \theta_{0}}{\partial Y}+u_{1} \frac{\partial \theta_{0}}{\partial x}+v_{1} \frac{\partial \theta_{0}}{\partial y}+w_{1} \frac{\partial \theta_{0}}{\partial z}+u_{0} \frac{\partial \theta_{1}}{\partial x}+v_{0} \frac{\partial \theta_{1}}{\partial y}+w_{0} \frac{\partial \theta_{1}}{\partial z}\right)+\frac{\partial p_{1}}{\partial Y}\right] \\
\frac{\partial u_{2}}{\partial x}+\frac{\partial v_{2}}{\partial y}+\frac{\partial w_{2}}{\partial z}=-\left(\frac{\partial u_{1}}{\partial X_{1}}+\frac{\partial u_{0}}{\partial X_{2}}+\frac{\partial v_{1}}{\partial Y}\right) .
\end{array}\right.
$$

Let

$$
\begin{aligned}
A u_{2}= & -\left[\frac{\partial u_{0}}{\partial T_{2}}+U \frac{\partial u_{0}}{\partial X_{2}}+U \frac{\partial u_{1}}{\partial X_{1}}+V \frac{\partial u_{1}}{\partial Y}+\frac{\partial u_{1}}{\partial T_{1}}\right. \\
+ & \frac{1}{\rho_{s}}\left(u_{0} \frac{\partial u_{0}}{\partial X_{1}}+v_{0} \frac{\partial u_{0}}{\partial Y}+u_{1} \frac{\partial u_{0}}{\partial x}+v_{1} \frac{\partial u_{0}}{\partial y}\right. \\
+ & \left.w_{1} \frac{\partial u_{0}}{\partial z}+u_{0} \frac{\partial u_{1}}{\partial x}+v_{0} \frac{\partial u_{1}}{\partial y}+w_{0} \frac{\partial u_{1}}{\partial z}\right)+\frac{\partial p_{0}}{\partial X_{2}} \\
+ & \left.\frac{\partial p_{1}}{\partial X_{1}}\right], \\
A v_{2} & =-\left[\frac{\partial v_{0}}{\partial T_{2}}+U \frac{\partial v_{0}}{\partial X_{2}}+U \frac{\partial v_{1}}{\partial X_{1}}+V \frac{\partial v_{1}}{\partial Y}+\frac{\partial v_{1}}{\partial T_{1}}\right. \\
+ & \frac{1}{\rho_{s}}\left(u_{0} \frac{\partial v_{0}}{\partial X_{1}}+v_{0} \frac{\partial v_{0}}{\partial Y}+u_{1} \frac{\partial v_{0}}{\partial x}+v_{1} \frac{\partial v_{0}}{\partial y}+w_{1} \frac{\partial v_{0}}{\partial z}\right. \\
+ & \left.\left.u_{0} \frac{\partial v_{1}}{\partial x}+v_{0} \frac{\partial v_{1}}{\partial y}+w_{0} \frac{\partial v_{1}}{\partial z}\right)+\frac{\partial p_{1}}{\partial Y}\right]
\end{aligned}
$$

$$
\begin{aligned}
A w_{2} & =-\left[\frac{\partial w_{0}}{\partial t_{1}}+U \frac{\partial w_{0}}{\partial X_{1}}+V \frac{\partial w_{0}}{\partial Y}+U \frac{\partial w_{1}}{\partial x}\right. \\
+ & \left.\frac{1}{\rho_{s}}\left(u_{0} \frac{\partial w_{1}}{\partial x}+v_{0} \frac{\partial w_{1}}{\partial y}+w_{0} \frac{\partial w_{1}}{\partial z}\right)+\frac{\partial w_{1}}{\partial t}\right], \\
A \theta_{2} & =-\left[\frac{\partial \theta_{0}}{\partial T_{2}}+U \frac{\partial \theta_{0}}{\partial X_{2}}+U \frac{\partial \theta_{1}}{\partial X_{1}}+V \frac{\partial \theta_{1}}{\partial Y}+\frac{\partial \theta_{1}}{\partial T_{1}}\right. \\
+ & \frac{1}{\rho_{s}}\left(u_{0} \frac{\partial \theta_{0}}{\partial x_{1}}+v_{0} \frac{\partial \theta_{0}}{\partial Y}+u_{1} \frac{\partial \theta_{0}}{\partial x}+v_{1} \frac{\partial \theta_{0}}{\partial y}+w_{1} \frac{\partial \theta_{0}}{\partial z}\right. \\
+ & \left.\left.u_{0} \frac{\partial \theta_{1}}{\partial x}+v_{0} \frac{\partial \theta_{1}}{\partial y}+w_{0} \frac{\partial \theta_{1}}{\partial z}\right)+\frac{\partial p_{1}}{\partial Y}\right] \\
A P_{2} & =-\left(\frac{\partial u_{1}}{\partial X_{1}}+\frac{\partial u_{0}}{\partial X_{2}}+\frac{\partial v_{1}}{\partial Y}\right) .
\end{aligned}
$$

Substitute (18) and (26) into (29) and collecting the secular-producing terms proportional to $e^{i\left(k_{j} x-\omega_{j} t\right)}$, we have 


$$
\begin{aligned}
& A u_{2}=\sum_{j=1}^{2} \widetilde{u_{0 j}}\left(A_{j T_{2}}+U A_{j X_{2}}\right)+U \widetilde{u_{1 j}} A_{j X_{1} X_{1}} \\
& +\frac{1}{\rho_{s}}\left(3 i k_{j} \widetilde{u_{0 j}} \widetilde{u_{1 j}}+\widetilde{u_{0 j}} \widetilde{v_{1 j}}+\widetilde{u_{0 j}} \widetilde{w_{1 j}}+\widetilde{v_{0 j}} \widetilde{u_{1 j}}\right. \\
& \left.+\widetilde{w_{0 j}} \widetilde{u_{1 j}}\right)\left|A_{j}\right|^{2} A_{j} \text {, } \\
& A v_{2}=\sum_{j=1}^{2} \widetilde{v_{0 j}}\left(A_{j T_{2}}+U A_{j X_{2}}\right)+V \widetilde{v_{1 j}} A_{j Y Y} \\
& +\frac{1}{\rho_{s}}\left(i k_{j} \widetilde{u_{1 j}} \widetilde{v_{0 j}}+\widetilde{v_{1}} \widetilde{v_{0 y}}+\widetilde{w_{1}} \widetilde{v_{0 z}}+2 i k_{j} \widetilde{u_{0}} \widetilde{v_{1}}\right. \\
& \left.+\widetilde{v_{0 j}} \widetilde{v_{1 j}}+\widetilde{w_{0 j}} \widetilde{v_{1 j}}\right)\left|A_{j}\right|^{2} A_{j}, \\
& A w_{2}=\sum_{j=1}^{2} \widetilde{w_{0 j}}\left(A_{j T_{1}}+U A_{j X_{1}}\right)+V \widetilde{w_{1}} A_{j Y Y}, \\
& A \theta_{2}=\sum_{j=1}^{2} \widetilde{\theta_{0 j}}\left(A_{j T_{2}}+U A_{j X_{2}}\right)+U \widetilde{\theta_{1}} A_{j X_{1} X_{1}} \\
& +\frac{1}{\rho_{s}}\left(i k_{j} \widetilde{u_{1 j}} \widetilde{\theta_{0 j}}+\widetilde{v_{1 j}} \widetilde{\theta_{0 j}}+2 i k \widetilde{u_{0 j}} \widetilde{\theta_{1 j}}+\widetilde{v_{0 j}} \widetilde{\theta_{1 j}}\right) \\
& \cdot\left|A_{j}\right|^{2} A_{j} \\
& A p_{2}=\sum_{j=1}^{2} \widetilde{u_{0 j}} A_{j X_{2}}+\widetilde{u_{1 j}} A_{j X_{1} X_{1}}+\widetilde{v_{1 j}} A_{j Y Y} .
\end{aligned}
$$

After eliminating other variables in (28), the equation about $p_{2}$ can be obtained as follows:

$$
\begin{aligned}
L_{y, z}\left(\frac{\partial p_{2}}{\partial x}\right)= & L_{1 y, z}\left(A u_{1}\right)+L_{2 y, z}\left(A v_{1}\right) \\
& +L_{3 y, z}\left(A w_{1}\right)+L_{4 y, z}\left(A \theta_{1}\right) .
\end{aligned}
$$

Introduce the following variables:

$$
\begin{aligned}
& x=\frac{1}{U} X_{1}=\frac{1}{U} X_{2}, \\
& t=T_{1}=T_{2} .
\end{aligned}
$$

There are conditions for solution of (31),

$$
\begin{aligned}
& i\left(\frac{\partial A_{1}}{\partial t}+\frac{\partial A_{1}}{\partial x}\right)+\alpha_{1} \frac{\partial^{2} A_{1}}{\partial x^{2}}+\beta_{1} \frac{\partial^{2} A_{1}}{\partial y^{2}} \\
& \quad+\gamma_{1}\left(\left|A_{1}\right|^{2}+\left|A_{2}\right|^{2}\right) A_{1}=0, \\
& i\left(\frac{\partial A_{2}}{\partial t}+\frac{\partial A_{2}}{\partial x}\right)+\alpha_{1} \frac{\partial^{2} A_{2}}{\partial x^{2}}+\beta_{1} \frac{\partial^{2} A_{2}}{\partial y^{2}} \\
& \quad+\gamma_{1}\left(\left|A_{1}\right|^{2}+\left|A_{2}\right|^{2}\right) A_{2}=0,
\end{aligned}
$$

where

$$
\begin{aligned}
\alpha_{1} & =-i\left(U \widetilde{u_{1 j}}+\widetilde{u_{1 j}}\right), \\
\beta_{1} & =-i\left(V \widetilde{v_{1 j}}+V \widetilde{w_{1 j}}+\widetilde{v_{1 j}}\right) \\
\gamma_{1} & =\frac{1}{\rho_{s}}\left[3 k_{j} \widetilde{u_{0 j}} \widetilde{u_{1 j}}+k \widetilde{u_{1 j}} \widetilde{v_{0 j}}+2 k \widetilde{u_{0 j}} \widetilde{v_{1 j}}-i\left(\widetilde{u_{0 j}} \widetilde{v_{1 j}}\right.\right. \\
& +\widetilde{u_{0 j}} \widetilde{w_{1 j}}+\widetilde{v_{0}} \widetilde{u_{1 j}}+\widetilde{w_{0}} \widetilde{u_{1 j}}+\widetilde{v_{0 j}} \widetilde{v_{1 j}}+\widetilde{w_{1 j}} \widetilde{v_{0 j}} \\
& \left.\left.+\widetilde{v_{0 j}} \widetilde{v_{1 j}}\right)\right] .
\end{aligned}
$$

\section{The Solutions of the Three-Dimensional CNLS Equations Group}

In the past, Hui [38] gave a solitary wave solution of $(2+1)$ dimensional nonlinear Schrödinger equation with the help of a space coordinate transformation. In this chapter, this method will be applied. Based on trial function method, we will discuss the solution of the CNLS equations group. The equations group is

$$
\begin{aligned}
& i \frac{\partial A_{1}}{\partial T}+\alpha_{1} \frac{\partial^{2} A_{1}}{\partial X^{2}}+\beta_{1} \frac{\partial^{2} A_{1}}{\partial Y^{2}} \\
& +\left(\sigma_{1} *\left|A_{1}\right|^{2}+r_{12} *\left|A_{2}\right|^{2}\right) A_{1}=0, \\
& i \frac{\partial A_{2}}{\partial T}+\alpha_{2} \frac{\partial^{2} A_{2}}{\partial X^{2}}+\beta_{2} \frac{\partial^{2} A_{2}}{\partial Y^{2}} \\
& \quad+\left(\sigma_{2} *\left|A_{2}\right|^{2}+r_{21} *\left|A_{1}\right|^{2}\right) A_{2}=0 .
\end{aligned}
$$

If we further introduce

$$
Z=X \cos (\theta)+Y \cos (\theta)=K X+M Y
$$

(35) reduces to

$$
\begin{aligned}
& i \frac{\partial A_{1}}{\partial T}+\gamma_{1} \frac{\partial^{2} A_{1}}{\partial Z^{2}}+\left(\sigma_{1} *\left|A_{1}\right|^{2}+r_{12} *\left|A_{2}\right|^{2}\right) A_{1}=0, \\
& i \frac{\partial A_{2}}{\partial T}+\gamma_{2} \frac{\partial^{2} A_{2}}{\partial Z^{2}}+\left(\sigma_{2} *\left|A_{2}\right|^{2}+r_{21} *\left|A_{1}\right|^{2}\right) A_{2}=0,
\end{aligned}
$$

where

$$
\begin{aligned}
& \gamma_{1}=\alpha_{1} \cos ^{2} \theta+\beta_{1} \sin ^{2} \theta, \\
& \gamma_{2}=\alpha_{2} \cos ^{2} \theta+\beta_{2} \sin ^{2} \theta .
\end{aligned}
$$

By rescaling the spatial coordinate and both amplitudes, we can reduce the number of nontrivial coefficients of the CNLS system; the simplified system is

$$
\begin{gathered}
i A_{T}+A_{\xi \xi}+|A|^{2} A+\widetilde{\gamma_{12}}|B|^{2} A=0, \\
i B_{T}+\gamma B_{\xi \xi}+|B|^{2} B+\widetilde{\gamma_{21}}|A|^{2} B=0,
\end{gathered}
$$


where the new unknowns and coefficients are

$$
\begin{aligned}
A & \equiv \sqrt{\sigma_{1}} A_{1}, \\
B & \equiv \sqrt{\sigma_{2}} A_{2}, \\
\xi & \equiv \sqrt{\frac{1}{\gamma_{1}}} Z, \\
\gamma & =\frac{\gamma_{1}}{\gamma_{2}}, \\
\widetilde{\gamma_{12}} & =\frac{\gamma_{12}}{\sigma_{2}}, \\
\widetilde{\gamma_{21}} & =\frac{\gamma_{21}}{\sigma_{1}} .
\end{aligned}
$$

The steadily oscillating solutions of this are solutions and their spatially periodical generalizations. Solitons and cnoidal waves are obtained by assuming

$$
\begin{aligned}
& A_{1}=\exp \left(i \mu^{2} \gamma_{1} t\right) u(Z), \\
& A_{2}=\exp \left(i \rho^{2} \gamma_{2} t\right) v(Z),
\end{aligned}
$$

where $\mu, \rho$ and $u, v$ are assumed to be real. Note that, in spite of the cross-modulation, the frequencies in the two modes are generally different. Substitute (41) into (39), a set of two coupled ODES equations' group can obtained and can be called SCNLS equations group

$$
\begin{aligned}
& u_{Z Z}-\mu^{2} u+\frac{\sigma_{1}}{\gamma_{1}} u^{3}+\frac{\gamma_{12}}{\gamma_{1}} u v^{2}=0, \\
& v_{Z Z}-\rho^{2} v+\frac{\sigma_{2}}{\gamma_{2}} v^{3}+\frac{\gamma_{21}}{\gamma_{2}} u^{2} v=0 .
\end{aligned}
$$

By rescaling the spatial coordinate and both amplitudes, it is possible to reduce the number of nontrivial coefficients of the SCNLS system from six to three. The simplified system is

$$
\begin{array}{r}
U_{\eta \eta}-U+2 U^{3}+\widetilde{\gamma_{12}} V^{2} U=0, \\
V_{\eta \eta}-\epsilon^{2} V+2 V^{3}+\widetilde{\gamma_{21}} U^{2} V=0,
\end{array}
$$

where the new unknowns and coefficients are

$$
\begin{aligned}
U & =\frac{1}{\mu} \sqrt{\frac{\sigma_{1}}{2 \gamma_{1}}} u, \\
V & =\frac{1}{\mu} \sqrt{\frac{\sigma_{2}}{2 \gamma_{2}}} v, \\
\epsilon & =\frac{\rho}{\mu}, \\
\widetilde{\gamma_{12}} & =\frac{2 \gamma_{2} \gamma_{12}}{\gamma_{1} \sigma_{2}}, \\
\widetilde{\gamma_{21}} & =\frac{2 \gamma_{1} \gamma_{21}}{\gamma_{2} \sigma_{1}} .
\end{aligned}
$$

To compute the solitary and cnoidal waves, it is always sufficient to solve this system of two ODES. However, to collide solitary waves it is necessary to shift the steadily oscillating solitary waves into a frame of reference such that they move at some translational velocity $V$.

If $A_{1}(X, T), A_{2}(X, T)$ are solutions to the CNLS equations group

$$
\begin{aligned}
& i \frac{\partial A_{1}}{\partial T}+\gamma_{1} \frac{\partial^{2} A_{1}}{\partial Z^{2}}+\left(\sigma_{1} *\left|A_{1}\right|^{2}+r_{12} *\left|A_{2}\right|^{2}\right) A_{1}=0, \\
& i \frac{\partial A_{2}}{\partial T}+\gamma_{2} \frac{\partial^{2} A_{2}}{\partial Z^{2}}+\left(\sigma_{2} *\left|A_{2}\right|^{2}+r_{21} *\left|A_{1}\right|^{2}\right) A_{2}=0,
\end{aligned}
$$

the another solution is

$$
\begin{aligned}
& \widetilde{A_{1}}=A_{1}(Z-V T) \exp \left(i \eta_{1} Z\right) \exp \left(-i \gamma_{1} Z_{1}^{2} T\right), \\
& \widetilde{A_{2}}=A_{2}(Z-V T) \exp \left(i \eta_{2} Z\right) \exp \left(-i \gamma_{2} Z_{2}^{2} T\right),
\end{aligned}
$$

where the velocity $V$ is

$$
V=2 \gamma_{1} \eta_{1}=2 \gamma_{2} \eta_{2},
$$

which implies that the constants $\xi_{1}$ and $\xi_{2}$ must be proportional as indicated set $\mu=\rho$ and define

$$
\begin{aligned}
& u=\theta_{1} g\left(Z, \mu^{2}\right) \\
& v=\theta_{2} g\left(Z, \mu^{2}\right),
\end{aligned}
$$

where $g(Z, c)$ is a solution to

$$
g_{Z Z}-\mu^{2} g+2 g^{3}=0
$$

Substitute (48) into the ODE system (42) and collecting terms in $g^{3}$ and imposing the condition that the coefficients must be equal 2 , the $2 \times 2$ matrix system is obtained

$$
\left(\begin{array}{ll}
\frac{\sigma_{1}}{\gamma_{1}} & \frac{\gamma_{12}}{\gamma_{1}} \\
\frac{\gamma_{21}}{\gamma_{2}} & \frac{\sigma_{2}}{\gamma_{2}}
\end{array}\right)\left(\begin{array}{l}
\theta_{1}^{2} \\
\theta_{2}^{2}
\end{array}\right)=\left(\begin{array}{l}
2 \\
2
\end{array}\right) .
$$

The solution is

$$
\begin{aligned}
& \theta_{1}^{2}=2 \frac{\gamma_{1} \sigma_{2}-\gamma_{2} \gamma_{12}}{\sigma_{1} \sigma_{2}-\gamma_{12} \gamma_{21}}, \\
& \theta_{2}^{2}=2 \frac{\gamma_{2} \sigma_{1}-\gamma_{1} \gamma_{21}}{\sigma_{1} \sigma_{2}-\gamma_{12} \gamma_{21}} .
\end{aligned}
$$

The limiting solitary waves for both branches are

$$
g= \pm \mu \operatorname{sech}(\mu Z) \text {. }
$$

So the solution of CNLS equations group is

$$
\begin{aligned}
& A_{1}=\sqrt{2 \frac{\gamma_{1} \sigma_{2}-\gamma_{2} \gamma_{12}}{\sigma_{1} \sigma_{2}-\gamma_{12} \gamma_{21}}} \mu \exp \left(i \mu^{2} \gamma_{1} t\right) \operatorname{sech}(\mu Z), \\
& A_{2}=\sqrt{2 \frac{\gamma_{2} \sigma_{1}-\gamma_{1} \gamma_{21}}{\sigma_{1} \sigma_{2}-\gamma_{12} \gamma_{21}}} \mu \exp \left(i \mu^{2} \gamma_{2} t\right) \operatorname{sech}(\mu Z),
\end{aligned}
$$

where $\sigma_{1}^{*}, \sigma_{2}^{*}$ are Landau coefficients and $\gamma_{12}^{*}, \gamma_{21}^{*}$ are interaction coefficients. 


\section{Energy Variation Characteristics of Coupled Envelope Gravity Waves}

In this section we will discuss two waves energy variation characteristics in the process of interaction; the first equation of (33) is multiplied by $A_{1}^{*}$; the conjugate equation is multiplied by $A_{1}$, subtracting the two types. In the same way, the second equation of (33) is multiplied by $A_{2}^{*}$; the conjugate equation is multiplied by $A_{2}$, subtracting the two types, we can obtain the following equations group:

$$
\begin{gathered}
i \frac{\partial}{\partial T}\left|A_{1}\right|^{2}+\alpha_{1} \frac{\partial}{\partial X}\left(A_{1}^{*} \frac{\partial A_{1}}{\partial X}-A_{1} \frac{\partial A_{1}^{*}}{\partial X}\right) \\
+\beta_{1} \frac{\partial}{\partial Y}\left(A_{1}^{*} \frac{\partial A_{1}}{\partial Y}-A_{1} \frac{\partial A_{1}^{*}}{\partial Y}\right)=0 \\
i\left(\frac{\partial}{\partial T}\left|A_{2}\right|^{2}+C_{g 2} * \frac{\partial\left|A_{2}\right|^{2}}{\partial X}\right) \\
+\alpha_{2} \frac{\partial}{\partial Y}\left(A_{2}^{*} \frac{\partial A_{2}}{\partial X}-A_{2} \frac{\partial A_{2}^{*}}{\partial X}\right) \\
+\beta_{2} \frac{\partial}{\partial Y}\left(A_{2}^{*} \frac{\partial A_{2}}{\partial Y}-A_{2} \frac{\partial A_{2}^{*}}{\partial Y}\right)=0 .
\end{gathered}
$$

$X, Y$ is integrated from $-\infty$ to $+\infty$; if $X, Y \rightarrow \pm \infty$ and $\left|A_{1}\right| \rightarrow$ 0 , we can get

$$
\begin{aligned}
& \frac{\partial}{\partial T} \int_{-\infty}^{\infty} \int_{-\infty}^{\infty}\left|A_{1}\right|^{2} d X d Y=0, \\
& \frac{\partial}{\partial T} \int_{-\infty}^{\infty} \int_{-\infty}^{\infty}\left|A_{2}\right|^{2} d X d Y=0 .
\end{aligned}
$$

Equations (55) show that $E_{1}=\int_{-\infty}^{\infty} \int_{-\infty}^{\infty}\left|A_{1}\right|^{2} d X d Y$ and $E_{2}=$ $\int_{-\infty}^{\infty} \int_{-\infty}^{\infty}\left|A_{1}\right|^{2} d X d Y$ are time invariants.

Obviously $E_{1}$ and $E_{2}$ are energy of two waves. This suggests that the energy of waves is conserved, when the coefficients satisfy

$$
\begin{aligned}
\alpha_{1} & =\alpha_{2}, \\
\sigma_{1} * & =\sigma_{2} *=\gamma_{12} *=\gamma_{21} *,
\end{aligned}
$$

or

$$
\begin{aligned}
\alpha_{1} & =-\alpha_{2}, \\
\sigma_{1} * & =\sigma_{2} *=-\gamma_{12} *=-\gamma_{21} *
\end{aligned}
$$

Equation (33) has infinite conservation law. Except the two kinds of energy conversation $E_{1}$ and $E_{2}$ above, (33) have four movement invariants:

$$
\begin{aligned}
E_{3} & =\int_{-\infty}^{\infty}\left(A_{1} \frac{\partial A_{*}}{\partial X}+\frac{\gamma_{12} *}{\gamma_{21} *} A_{2} \frac{\partial A_{2}^{*}}{\partial X}\right) d X, \\
E_{4} & =\int_{-\infty}^{\infty}\left(A_{1} \frac{\partial A_{*}}{\partial Y}+\frac{\gamma_{12} *}{\gamma_{21} *} A_{2} \frac{\partial A_{2}^{*}}{\partial Y}\right) d Y, \\
E_{5} & =\int_{-\infty}^{\infty}\left(\alpha_{1}\left|\frac{\partial A_{1}}{\partial X}\right|^{2}+\frac{\gamma_{12} *}{\gamma_{21} *} \alpha_{2}\left|\frac{\partial A_{2}}{\partial X}\right|^{2}-\frac{\sigma_{1}^{*}}{2}\left|A_{1}\right|^{4}\right. \\
& \left.-\frac{\gamma_{12} *}{\gamma_{21} *} \frac{\sigma_{1}^{*}}{2}\left|A_{2}\right|^{4}-\gamma_{12}^{*}\left|A_{1}\right|^{2}\left|A_{2}\right|^{2}\right) d X, \\
E_{6} & =\int_{-\infty}^{\infty}\left(\alpha_{1}\left|\frac{\partial A_{1}}{\partial Y}\right|^{2}+\frac{\gamma_{12} *}{\gamma_{21} *} \alpha_{2}\left|\frac{\partial A_{2}}{\partial Y}\right|^{2}-\frac{\sigma_{1}^{*}}{2}\left|A_{1}\right|^{4}\right. \\
& \left.-\frac{\gamma_{12} *}{\gamma_{21} *} \frac{\sigma_{1}^{*}}{2}\left|A_{2}\right|^{4}-\gamma_{21}^{*}\left|A_{1}\right|^{2}\left|A_{2}\right|^{2}\right) d Y .
\end{aligned}
$$

Equations (58) to (61) are the momentum of two waves, so we can confirm the conservation of momentum.

\section{Modulation Instability of a Uniform Gravity Waves Trains in 3-Dimension Space}

We will discuss the modulation instability for threedimensional gravity wave trains in this section. Based on (53) we can obtain the solution of a uniform wave train. Initial solution of the equation can take the following form:

$$
A=A_{0} \exp \left(i \sigma_{1} A_{0}^{2} T\right)
$$

according to the studies of Yuen and Lake [39], the perturbed wave trains may be expressed as follows:

$$
\begin{aligned}
A= & \left\{A_{0}+A_{+} \exp [i(K X+M Y+\omega T)]\right. \\
& \left.+A_{-} \exp \left[-i\left(K X+M Y+\omega^{*} T\right)\right]\right\} \exp \left(i \sigma_{1} A_{0}^{2} T\right),
\end{aligned}
$$

where, in fact, $A_{0}$ denotes the amplitude of a uniform disturbance stream function, $A_{ \pm}$is the amplitude of a sideband disturbance and is small enough compared with $A_{0}$ to permit linearization, $\omega$ is eigenvalue to be determined, and its conjugate is $\omega *$. Informed by Craik [40], $\pm K, \pm M$ denotes the prescribed zonal and meridional wave number perturbations in the $X$ and $Y$ directions. Here, we define $\delta( \pm K, \pm M)=( \pm k p, \pm m q)$, in which $p \ll 1$ and $q \ll 1$ are required. Linearization with respect to $A_{0}$ satisfied the following eigenvalue equation:

$$
\begin{aligned}
\omega^{2} & =\left(\alpha K^{2}+\beta M^{2}\right)\left(\alpha K^{2}+\beta M^{2}-2 \sigma_{1} A_{0}^{2}\right) \\
= & \frac{1}{\delta^{4}}\left\{\left[\alpha(p k)^{2}+\beta(q m)^{2}\right]\right. \\
& \left.\cdot\left[\alpha(p k)^{2}+\beta(q m)^{2}-2 \sigma_{1} \delta^{2} A_{0}^{2}\right]\right\} .
\end{aligned}
$$




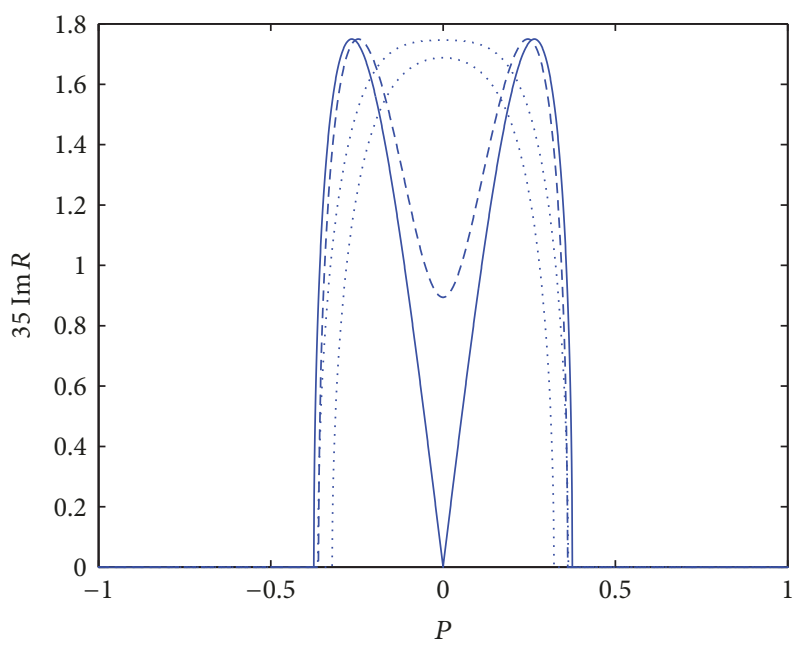

Figure 1: The solid curves denote the case of $q=0$, the dot curves $q=0.08$, and the dashed curves $q=0.24, \alpha=1, \beta=1$, and $\sigma_{1}=0.1$, $30^{\circ} \mathrm{N}$.

If $B_{0}=\delta A_{0}$ and $\omega^{2}=R^{2} / \delta^{4}$ are defined, we can get

$$
\begin{aligned}
R^{2}= & {\left[\alpha(p k)^{2}+\beta(q m)^{2}\right] } \\
& \cdot\left\{\left[\alpha(p k)^{2}+\beta(q m)^{2}\right]-2 \sigma_{1} B_{0}^{2}\right\}
\end{aligned}
$$

if $p$ and $q$ satisfy

$$
0<\alpha(p k)^{2}+\beta(q m)^{2}<2 \sigma_{1} B_{0}^{2},
$$

the uniform gravity wave train is unstable.

When $q=0$, the result resembles the instability diagram of Benjamin-Feir instability [41]. The case of $q \neq 0$ has not been studied yet. To obtain more detailed results, for gravity solitary waves, we only study the case where the fundamental number is 1 ; that is,

$$
k=\frac{1}{2.380 \cos \left(\phi_{0}\right)},
$$

where $\phi_{0}$ is the latitude. We will discuss the influence of $q$ and latitude value on the instability regions. Taking $L y=$ $6, n_{1}=2$ and $B_{0}=0.5$. In this case the instability growth rates $35 \operatorname{Im} R(35 \times \operatorname{Im} R)$ of a uniform gravity wave train at $30^{\circ} \mathrm{N}$, $45^{\circ} \mathrm{N}$, and $60^{\circ} \mathrm{N}$ for $q=0,0.08,0.24$ are shown in Figures 1,2 , and 3.

From Figure 1 we know that the instability region is $|p| \leq$ 0.38 for $q=0.08,|p| \leq 0.37$ for $q=0.08$, and $|p| \leq 0.30$ for $q=0.24$ at the latitude $30^{\circ} \mathrm{N}$. This shows that the instability region of $p$ will become narrower if $q$ increase in the small value limit. Meanwhile, at latitudes $45^{\circ} \mathrm{N}$ and $60^{\circ} \mathrm{N}$, there are the same properties as those at latitude $30^{\circ} \mathrm{N}$, as shown in Figures 2 and 3 .

In addition, as shown in the figures we can also note that the instability of $p$ will become wider if the latitude increases for the same $q$. It is worth noting that the higher the latitude, the more unstable the uniform gravity wave trains. This conclusion is valid for all small values of $q$. In this paper,

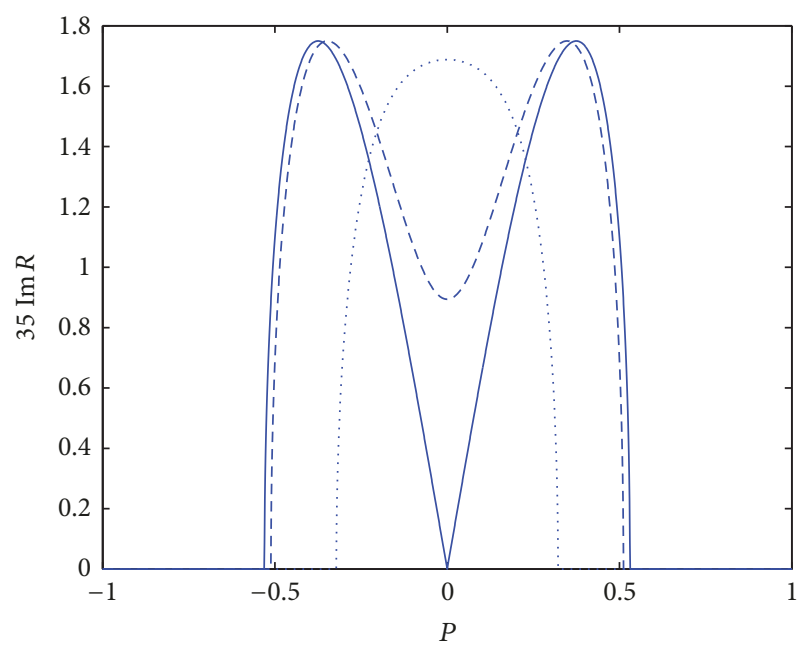

Figure 2: The solid curves denote the case of $q=0$, the dot curves $q=0.08$, and the dashed curves $q=0.24, \alpha=1, \beta=1$, and $\sigma_{1}=0.1$, $45^{\circ} \mathrm{N}$.

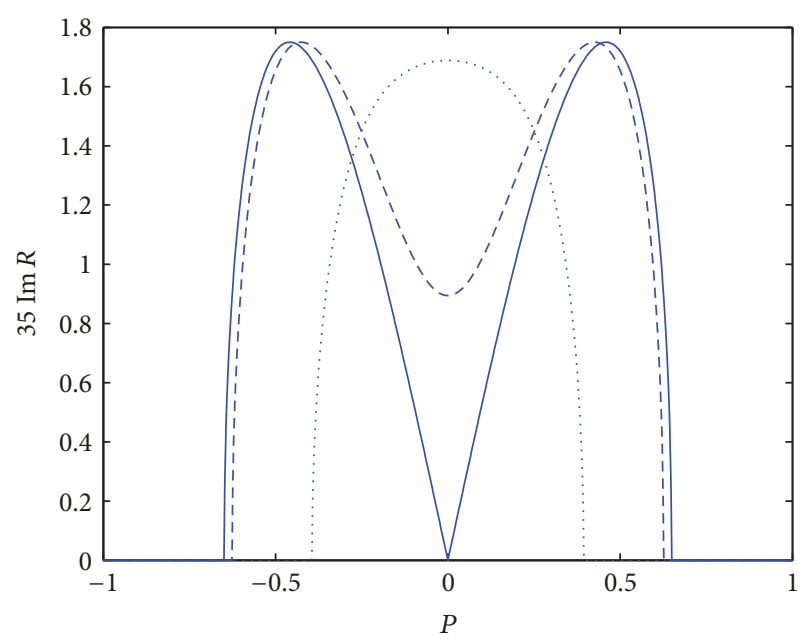

FIgURE 3: The solid curves denote the case of $q=0$, the dot curves $q=0.08$, and the dashed curves $q=0.24, \alpha=1, \beta=1$, and $\sigma_{1}=0.1$, $60^{\circ} \mathrm{N}$.

the effects of external forcing on the modulational instability of gravity wave trains are not discussed. This problem remains to be further studied.

\section{Conclusions}

In this paper, overcoming the limitations of calculations and using the appropriate method, we obtained a new model. Using the multiscale analysis and turbulence method, from the basic dynamic equations of multivariable in baroclinic environment, we derived out the gravity wave model, which is a coupled nonlinear Schrödinger equation (CNLS). Not only is the model three-dimensional and more suited to describe the feature of two envelope gravity solitary waves in a plane, specially, but also it is a coupled model and can show the interaction process between two waves. Based on 
the CNLS model, using the trial function method to solve the equations, the analytical solution was obtained. By observing the structures of the solution, the evolution characteristics of gravity solitary waves were obtained. Finally, modulational instability of a uniform three-dimensional gravity waves trains were also discussed. This shows that the instability region of $p$ will become narrower if $q$ increase in the small value limit. Meanwhile, at latitudes $45^{\circ} \mathrm{N}$ and $60^{\circ} \mathrm{N}$, there are the same properties as those at latitude $30^{\circ} \mathrm{N}$. In this paper, the effects of external forcing on the modulational instability of gravity wave trains are not discussed. This problem remains to be further studied.

\section{Conflicts of Interest}

The authors declare that there are no conflicts of interest regarding the publication of this paper.

\section{Acknowledgments}

This project is supported by National Key Research and Development Program (20175001722), Special Fund for Strategic Pilot Technology Chinese Academy of Sciences (XDA11010203), China Postdoctoral Science Foundation Funded Project (2017M610436), and Open Fund of the Key Laboratory of Meteorological Disaster of Ministry of Education (Nanjing University of Information Science and Technology) (KLME1507).

\section{References}

[1] J. S. Russell, "Repeat on waves," Tech. Rep. 41, British Association for the Advancement of Science, 1837.

[2] R. R. Long, "Solitary waves in the westerlies," Journal of the Atmosphere Sciences, vol. 21, pp. 197-200, 1964.

[3] D. J. Benny, "Long non-linear waves in fluid flows," Studies in Applied Mathematics, vol. 45, pp. 52-63, 1966.

[4] H. Yang, Q. Zhao, B. Yin, and H. Dong, "A new integrodifferential equation for rossby solitary waves with topography effect in deep rotational fluids," Abstract and Applied Analysis, vol. 2013, Article ID 597807, 2013.

[5] H. Yang, B. Yin, Y. Shi, and Q. Wang, "Forced ILW-Burgers equation as a model for Rossby solitary waves generated by topography in finite depth fluids," Journal of Applied Mathematics, vol. 2012, Article ID 491343, 2012.

[6] M. C. Li, "Nonlinear processes of squall line formation in the atmosphere and KdV equations," Scientia Sinica, vol. 11, pp. 341350, 1981.

[7] S. D. Liu and S. K. Liu, "The solution of nonlinear wave equation in atmosphere," Acta Meteorologica Sinica, vol. 40, pp. 279-288, 1982.

[8] D. H. Luo, "On the nonlinear Benjamin-Ono equation in atmosphere and its generalization," Science in China (Series B), vol. 32, pp. 1233-1245, 1989.

[9] Y. Shi, B. Yin, H. Yang, D. Yang, and Z. Xu, "Dissipative nonlinear Schrödinger Equation for envelope solitary Rossby waves with dissipation effect in stratified fluids and its solution," Abstract and Applied Analysis, Article ID 643652, Art. ID 643652, 9 pages, 2014.
[10] W. X. Ma, “Trigonal curves and algebro-geometric solution to soliton hierarchies I," Proceedings of the Royal Society A, vol. 473, Article ID 20170232, 2017.

[11] W. X. Ma, "Trigonal curves and algebro-geometric solution to soliton hierarchies II," Proceedings of the Royal Society A, vol. 473, Article ID 20170233, 2017.

[12] Y. J. Cui, "Uniqueness of solution for boundary value problems for fractional differential Equations," Applied Mathematics Letters, vol. 51, pp. 48-54, 2016.

[13] Z. Bai, X. Dong, and C. Yin, "Existence results for impulsive nonlinear fractional differential equation with mixed boundary conditions," Boundary Value Problems, vol. 1, pp. 63-71, 2016.

[14] H. Li, "Existence of nontrivial solutions for unilaterally asymptotically linear three-point boundary value problems," Abstract and Applied Analysis, vol. 2014, Article ID 263042, 7 pages, 2014.

[15] X.-X. Xu, "An integrable coupling hierarchy of the MkdvIntegrable systems, its hamiltonian structure and corresponding nonisospectral integrable hierarchy," Applied Mathematics and Computation, vol. 216, no. 1, pp. 344-353, 2010.

[16] H.-X. Yang, J. Du, X.-X. Xu, and J.-P. Cui, "Hamiltonian and super-Hamiltonian systems of a hierarchy of soliton equations," Applied Mathematics and Computation, vol. 217, no. 4, pp. 14971508, 2010.

[17] H. Dong, K. Zhao, H. Yang, and Y. Li, "Generalised (2+1)dimensional super MKdV hierarchy for integrable systems in soliton theory," East Asian Journal on Applied Mathematics, vol. 5, no. 3, pp. 256-272, 2015.

[18] B. Feng, Y. Zhang, and H. Dong, "A few integrable couplings of some integrable systems and $(2+1)$-dimensional integrable hierarchies," Abstract and Applied Analysis, vol. 2014, Article ID 932672, 2014.

[19] X. Guo, "On bilinear representations and infinite conservation laws of a nonlinear variable-coefficient equation," Applied Mathematics and Computation, vol. 248, pp. 531-535, 2014.

[20] T. Xu and Y. Chen, "Localized waves in three-component coupled nonlinear Schroodinger equation," Chinese Physics B, vol. 25, Article ID 090201, 2016.

[21] Y. Li, W.-X. Ma, and J. E. Zhang, "Darboux transformations of classical Boussinesq system and its new solutions," Physics Letters A, vol. 275, no. 1-2, pp. 60-66, 2000.

[22] Q.-L. Zhao, X.-Y. Li, and F.-S. Liu, "Two integrable lattice hierarchies and their respective Darboux transformations," Applied Mathematics and Computation, vol. 219, no. 10, pp. 5693-5705, 2013.

[23] D.-y. Chen, D.-j. Zhang, and S.-f. Deng, "The novel multisoliton solutions of the MKdV-sine Gordon equations," Journal of the Physical Society of Japan, vol. 71, no. 2, pp. 658-659, 2002.

[24] E. G. Fan and H. Q. Zhang, "The homogeneous balance method for nonlinear soliton equations," Acta Physics Sinica, vol. 47, pp. 353-362, 1998.

[25] S. Liu, Z. Fu, S. Liu, and Q. Zhao, "Jacobi elliptic function expansion method and periodic wave solutions of nonlinear wave equations," Physics Letters A, vol. 289, no. 1-2, pp. 69-74, 2001.

[26] W. X. Ma and R. G. Zhang, "Adjont symmetry constraints of muliti-component AKNS equations," Chinese Anals of Mathematics, vol. 23, pp. 373-384, 2002.

[27] H. W. Yang, Z. H. Xu, D. Z. Yang, X. R. Feng, B. S. Yin, and H. H. Dong, "ZK-Burgers equation for three-dimensional Rossby solitary waves and its solutions as well as chirp effect," Advances in Difference Equations, 2016:167, 22 pages, 2016. 
[28] K. C. Le and L. T. K. Nguyen, Energy Methods in Dynamics, Springer Verlag, Berlin, Germany, 2014.

[29] K. C. Le and L. T. Nguyen, "Slope modulation of waves governed by sine-Gordon equation," Communications in Nonlinear Science and Numerical Simulation, vol. 18, no. 7, pp. 1563-1567, 2013.

[30] K. C. Le and L. T. Nguyen, "Amplitude modulation of waves governed by Korteweg-de Vries equation," International Journal of Engineering Science, vol. 83, pp. 117-123, 2014.

[31] K. C. Le and L. T. K. Nguyen, "Slope modulation of ring waves governed by two-dimensional sine-Gordon equation," Wave Motion, vol. 55, pp. 84-88, 2015.

[32] Q.-L. Zhao and X.-Y. Li, "A Bargmann system and the involutive solutions associated with a new 4-order lattice hierarchy," Analysis and Mathematical Physics, vol. 6, no. 3, pp. 237-254, 2016.

[33] X.-Y. Li, Y.-X. Li, and H.-X. Yang, "Two families of Liouville integrable lattice equations," Applied Mathematics and Computation, vol. 217, no. 21, pp. 8671-8682, 2011.

[34] A. Biswas, "1-Soliton solution of the generalized ZakharovKuznetsov equation with nonlinear dispersion and timedependent coefficients," Physics Letters A, vol. 373, no. 33, pp. 2931-2934, 2009.

[35] C. M. Khalique and G. Magalakwe, "Combined sinh-coshGordon equation: Symmetry reductions, exact solutions and conservation laws," Quaestiones Mathematicae, vol. 37, no. 2, pp. 199-214, 2014.

[36] K. C. Le and L. T. K. Nguyen, "Amplitude modulation of water waves governed by Boussinesq's equation," Nonlinear Dynamics, vol. 81, no. 1-2, pp. 659-666, 2015.

[37] R. Zhang, L. Yang, J. Song, and H. Yang, "(2+1) dimensional Rossby waves with complete Coriolis force and its solution by homotopy perturbation method," Computers \& Mathematics with Applications, vol. 73, no. 9, pp. 1996-2003, 2017.

[38] W. H. Hui, "Exact envelope-soliton solutions of a twodimensional nonlinear wave equation," Zeitschrift für Angewandte Mathematik und Physik (ZAMP), vol. 30, no. 6, pp. 929936, 1979.

[39] H. C. Yuen and B. M. Lake, "Nonlinear Dynamics of DeepWater Gravity Waves," Advances in Applied Mechanics, vol. 22, no. C, pp. 67-229, 1982.

[40] A. D. D. Craik, Wave Interactions and Fluid Flows, Cambridge University Press, London, UK, 1985.

[41] H. C. Yuen and W. E. Ferguson Jr., "Relationship between Benjamin-Feir instability and recurrence in the nonlinear Schrödinger equation," Physics of Fluids, vol. 21, no. 8, pp. 12751278, 1978. 


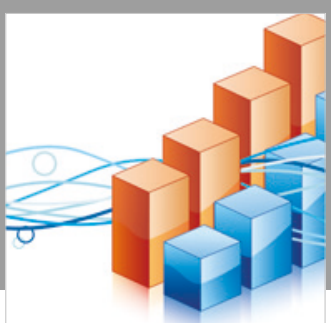

Advances in

Operations Research

\section{-n-m}
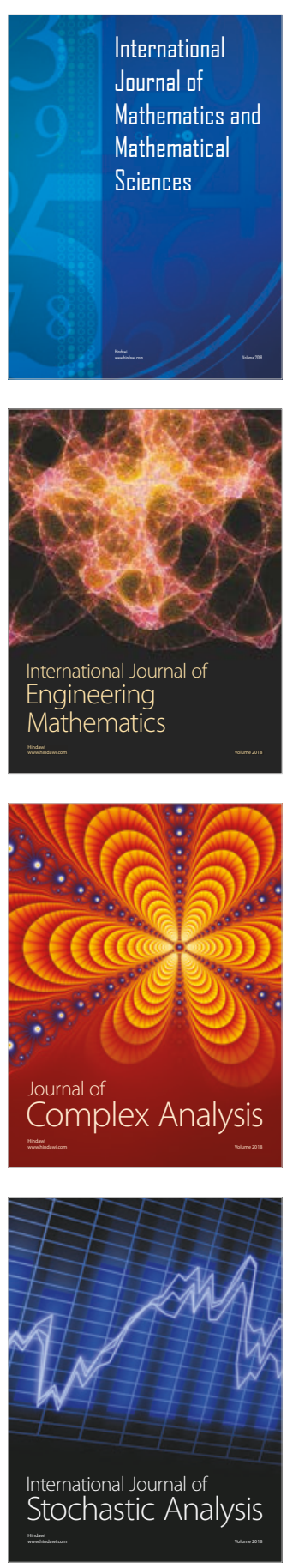
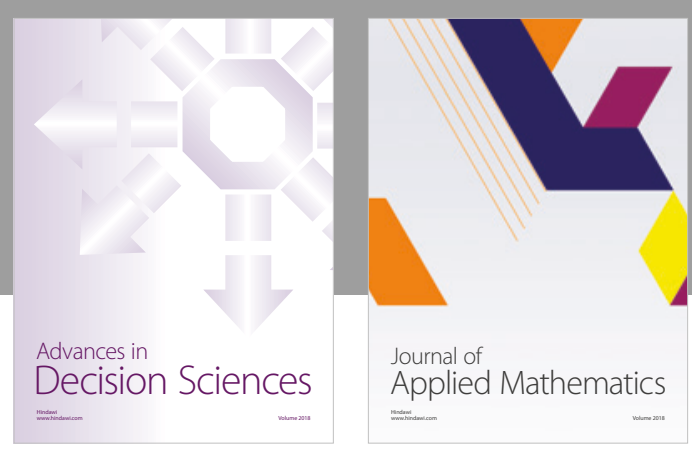

Journal of

Applied Mathematics
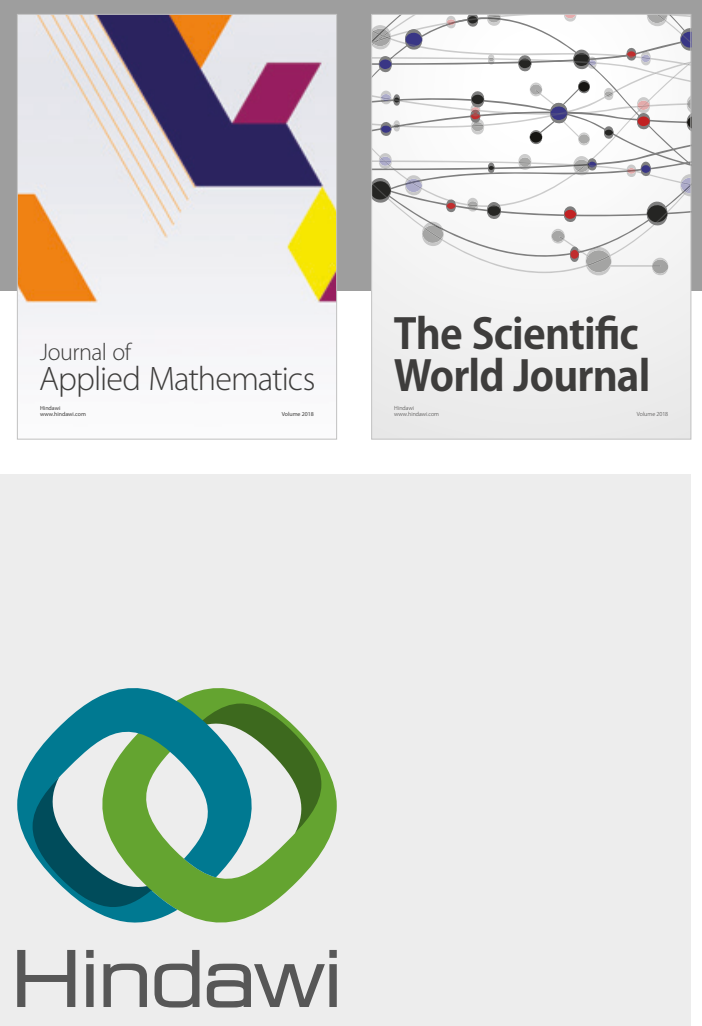

Submit your manuscripts at

www.hindawi.com

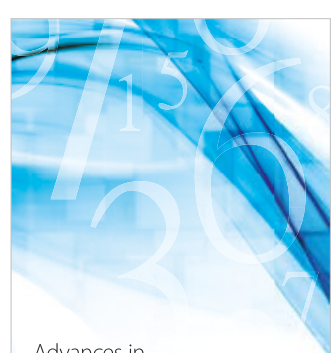

Advances in
Numerical Analysis
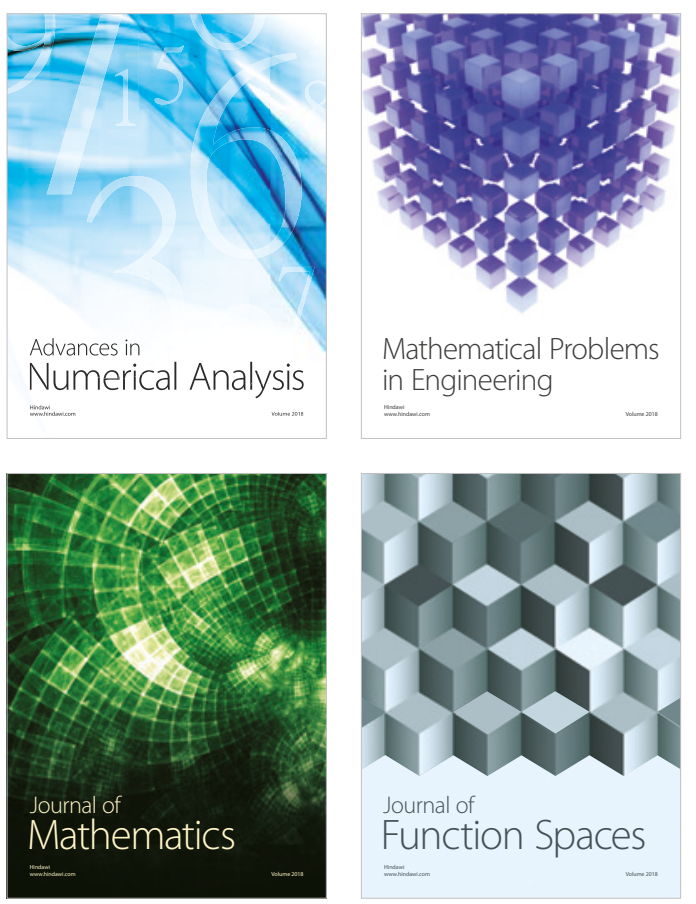

Mathematical Problems in Engineering

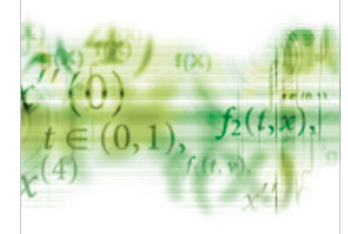

International Journal of

Differential Equations

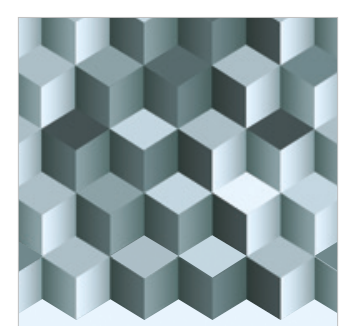

Journal of

Function Spaces

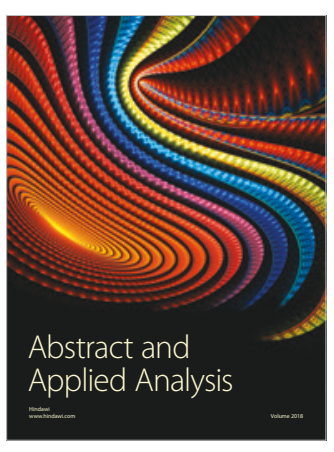

The Scientific

World Journal

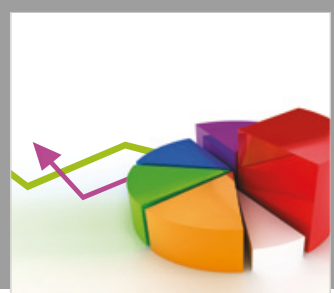

Journal of

Probability and Statistics
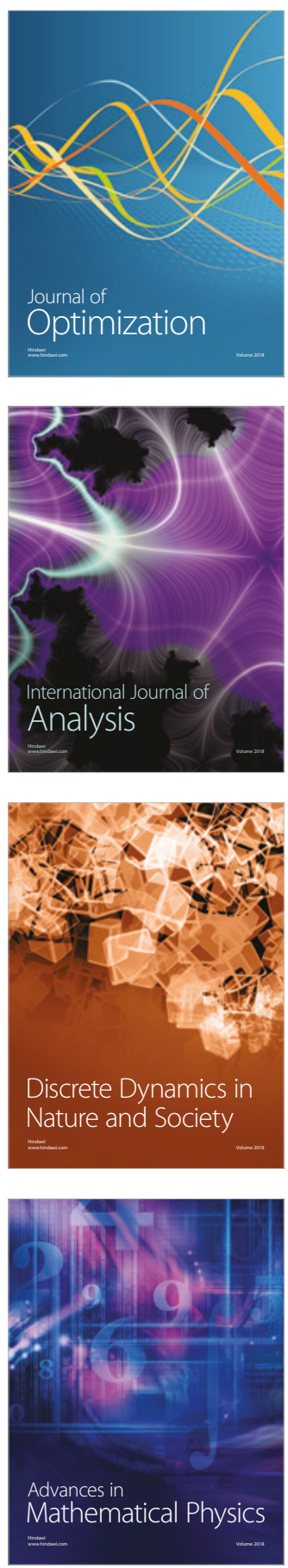